\title{
Analysis of effective surface area for electrochemical reaction derived from mass transport property
}

\author{
Hiroshi Iden ${ }^{\mathrm{a}}$, Anthony R. Kucernak ${ }^{\mathrm{b}}$ \\ ${ }^{a}$ Nissan Research Center, Nissan Motor Co., Ltd., Yokosuka, Kanagawa 237-8523, Japan \\ ${ }^{\mathrm{b}}$ Department of Chemistry, Imperial College London, London SW7 2AZ, United \\ Kingdom
}

\begin{abstract}
The effective surface area, $S^{\text {eff }}$, for electrochemical reaction at a Pt electrode was analyzed using mass transport property as a probe. This parameter is different to the socalled electrochemical surface area (ECA), which is usually obtained from the amount of protons which are underpotentially deposited, $H_{U P D}$, or by $\mathrm{CO}$ stripping. The analysis was done using both experimental and numerical methods. In experiments, a rotating disk electrode (RDE) was used under the conditions where the Pt surface can be partially covered by $\mathrm{Pt}$ oxides or CO. For numerical analysis, three mass transport models based on diffusion, convection and convective-diffusion were used. As a result, it was found that it is possible to estimate $S^{\text {eff }}$ from the change in Koutecky-Levich slope at high rotation speeds. The analysis suggests that $S^{\text {eff }}$ does decrease notably from relatively low potential (around $0.4 \mathrm{~V}$ ), even though it is not obvious in measured currents as the effect is masked by the low rates of mass transport. The analysis also suggests that there is a large difference in $S^{\text {eff }}$ with different methods, namely, estimation from the amount of $\mathrm{Pt}$ oxides and the analysis derived from mass transport property.
\end{abstract}

Keywords: Effective surface area; RDE; HOR; catalyst

Research highlights

- Estimation of effective surface area was possible by analyzing mass transport.

- Inactive domain size could also be estimated from the analysis.

- Effectiveness decreased notably from relatively low potential unlike Pt oxide growth.

\section{Introduction}

It is well known that a polymer electrolyte fuel cell (PEFC) is a promising primary power source for automotive use, and car manufacturers are developing fuel cell electric vehicles (FCEVs). However, there are still challenges to be addressed for the commercialization, such as power density, durability, sub-zero startup and cost reductions. From an industrial point of view, the most important aspect is cost reduction. According to recent DOE studies, Pt used as a catalyst accounts for about a quarter of the total fuel system cost [1]. Therefore, increasing the utilization of platinum in fuel cells is an important strategy in reducing total system cost.

In order to make the most of Pt, carbon supports with high surface areas (HSA carbon) and various core-shell catalysts have been developed. With HSA carbon supports, smaller Pt particles can be deposited densely, resulting in high surface area and high mass-specific activity $[2,3]$. On the other hand, core-shell catalysts reduce Pt usage by replacing the core of a Pt particle, which does not directly contribute to electrochemical reactions, with other less expensive materials $[4,5]$. In either approach, it is necessary to 
evaluate how much surface area contributed to electrochemical reactions under practical FCEV operation conditions.

Usually, the electrochemical surface area (ECA) of $\mathrm{Pt}$ is evaluated from the amount of protons which are underpotentially deposited, $H_{U P D}$, or by $\mathrm{CO}$ stripping [6]. However, the values obtained with those methods may not be applicable to practical operation conditions, for example, at high potentials (over $0.7 \mathrm{~V}$ ). The oxygen reduction reaction (ORR) current may be modeled by considering the ORR far from the equilibrium potential having a certain platinum site dependency in which site interactions are handled by a Temkin term and expressed as

$i_{k}=n F k C_{O_{2}}(1-\theta)^{x} \exp \left(-\frac{\beta F \eta}{R T}\right) \exp \left(-\frac{\gamma r \theta}{R T}\right)$ (1) [7],

where $n$ is the number of electrons, $F$ is the Faraday constant, $k$ is the rate constant, $C_{\mathrm{O}_{2}}$ is the concentration of $\mathrm{O}_{2}$ in the solution, $\theta$ is the total surface coverage by adsorbed species, $x$ is the number of $\mathrm{Pt}$ sites occupied by the adsorbate, $\beta$ and $\gamma$ are the symmetry factors and $r$ is the Temkin parameter. As can be seen in Eq. (1), the ORR current strongly depends on $\theta$. Although $\theta$ is sometimes evaluated by measuring the amount of Pt oxide [8,9], such an approach does not seem that straightforward for two reasons: the first is that we are interested in the number of free platinum sites $(1-\theta)$ and as a range of platinum oxides are formed as a function of potential, determining this parameter is difficult; the second is that the different oxides produced may interact with the ORR intermediates in different ways, that is $r$ is a function of $E$.

One of us has developed a method to evaluate the effective surface area, $S^{\text {eff }}$, for the hydrogen oxidation reaction (HOR) as a function of potential by using the change of mass transport properties as a probe [10]. This method is applicable to a membrane electrode assembly which has a gas diffusion layer on its catalyst layer. However, MEAs do not seem suitable for the analysis of the increase of $\theta$ at high potentials or validation of mitigation strategies for this issue. Therefore, it is desirable that this kind of technique is applied to a simpler system with which the analysis or the validation can be done more easily.

A rotating disk electrode (RDE) has been used to investigate fuel cell catalyst performance for many years [11-13]. The RDE is a useful tool for evaluating fuel cell catalyst performance because it allows extraction of the catalytic activity from the current density by control of the rotation speed. Moreover, it does not require as much catalyst material as an MEA. Furthermore MEA fabrication processes are not necessary for the RDE. However, in aqueous electrolytes such as $\mathrm{H}_{2} \mathrm{SO}_{4}$ or $\mathrm{HClO}_{4}$, the concentration and diffusion coefficient of oxygen and hydrogen are quite low. Thereby, the limiting currents are considerably lower than those which would be observed with actual catalyst layers [14]. In addition, the RDE cannot enhance the mass transport that much due to the cavitation of the electrolyte at rotation rates approaching $10000 \mathrm{rpm}$. Because of bubble formation due to cavitation, data analysis becomes significantly more complicated because the flow pattern of the solution is no longer laminar and become turbulent.

Regardless of the drawbacks, it was still thought to be useful to develop a similar method for evaluating $S^{\text {eff }}$ in a simpler system due to its feature of controllable mass transport. In this study, we investigated a new experimental method for evaluating $S^{\text {eff }}$ under the conditions where Pt surface can be partially covered. The validity of the method was examined by numerical approaches. 


\section{Experimental}

\subsection{Experimental setup}

All the electrochemical measurements were performed using a three-electrode electrochemical cell consisting of three compartments for a reference electrode (RE), a counter electrode (CE) and a working electrode (WE). A reversible hydrogen electrode (RHE) was employed as reference electrode, and all the potentials are referred to this electrode. A Pt wire served as a counter electrode. Before the electrochemical measurements, all glassware and electrodes were soaked in acidified potassium permanganate over $8 \mathrm{~h}$, rinsed with acidified hydrogen peroxide and then rinsed at least six times with ultra pure water (Millipore Milli-Q, $18.2 \mathrm{M} \Omega \mathrm{cm}$ ). Gas purities of $\geq 5.8 \mathrm{~N}$ (Air Products) were utilized with $6 \mathrm{~N}$ rated regulators (GCE DruVa). An aqueous $0.1 \mathrm{M}$ $\mathrm{HClO}_{4}$ solution was used as the electrolyte. The solution was prepared from $70 \% \mathrm{HClO}_{4}$ (Suprapur ${ }^{\circledR}$, Merck) and ultrapure water. Pt rotating disk electrodes with a diameter of 2 or $5 \mathrm{~mm}$ (AFE2M020PT or AFE2M050PT, PINE instruments) which are applicable up to $7000 \mathrm{rpm}$ were used as the WE. The RDE was attached to a modulated speed rotator (AFMSRCE, PINE instruments) and its rotation was controlled with the system while the potential was controlled by a Gamry Reference 600 potentiostat. All electrochemical experiments were conducted at room temperature.

\subsection{Conditioning of Pt RDE and roughness factor measurement}

Prior to the HOR measurements, the potential of the WE was electrochemically cleaned by cycling 100 times between 0 and $1.4 \mathrm{~V}^{\text {at }} 0.5 \mathrm{~V} \mathrm{~s}^{-1}$ in $0.1 \mathrm{M} \mathrm{HClO}_{4}$ saturated with $\mathrm{N}_{2}$ gas. After that, cyclic voltammetry was carried out to determine the roughness factor of the Pt disk. The potential of the WE was scanned between 0.02 and $1.2 \mathrm{~V}$ vs. $\mathrm{RHE}$ at a scan rate of $50 \mathrm{mV} \mathrm{s}^{-1}$ for several cycles. The amount of $H_{U P D}, Q_{H}$ was calculated by extracting the quantity of electricity due to hydrogen evolution reaction (HER) from total reduction current below the potential where the double layer region was observed. The detail was explained in Ref. 15. Then, the roughness factor was evaluated from $Q_{H}$, the geometrical area of the disk electrode and a charge to Pt surface area conversion factor of $210 \mu \mathrm{C} \mathrm{cm}_{\mathrm{Pt}}^{-2}$ for a smooth polycrystalline Pt.

\subsection{HOR measurement}

After the roughness factor measurement, the solution was saturated with $\mathrm{H}_{2}$. Hydrodynamic voltammograms for the HOR were obtained by cycling the potential from 0 to $1.1 \mathrm{~V}$ at $10 \mathrm{mV} \mathrm{s}^{-1}$ under the rotating rates of $400-6800 \mathrm{rpm}$. The conditions for HOR measurement is summarized in Table 1. 
Table 1

Experimental conditions for HOR measurement with the RDE and the MEA

\begin{tabular}{lcc}
\hline & RDE & MEA* \\
\hline Catalyst & Pt disk & Pt/GKB (46 wt. \%) \\
Carbon support & - & $\begin{array}{c}\text { Graphitized ketjen black } \\
\text { Nafion }{ }^{\circledR}\end{array}$ \\
Electrolyte & $0.1 \mathrm{M} \mathrm{HClO}_{4}$ & (D2020, EW1000, DuPont) \\
Relative humidity & - & $90 \%$ \\
Potential range & $0 \sim 1.1 \mathrm{~V}$ & $0.95 \sim 0.2 \mathrm{~V}$ \\
Potential control & Cycle $(10 \mathrm{mV} / \mathrm{s})$ & Potential step \\
Sweep (or step) direction & Anodic and cathodic (cycle) & (holding time: 5 min. for each step) \\
Temperature & Room temperature & Cathodic \\
\hline
\end{tabular}

* Ref. 10.

\subsection{Partial blocking with carbon monoxide}

For comparison between the free $\mathrm{Pt}$ surface determined from integration of $\mathrm{Pt}$ oxide charge, the Pt disk was also partially blocked by carbon monoxide (CO). First, $\mathrm{CO}$ was bubbled into the solution for about 15 min whilst the electrode potential was held at $0.05 \mathrm{~V}$. Next, $\mathrm{CO}$ was purged with $\mathrm{N}_{2}$ for over 30 min while the potential of the WE was held at $0.05 \mathrm{~V}$ so as to keep the adsorbed CO still on the Pt surface. The potential of the Pt disk was then cycled once between 0.05 and $0.7 \mathrm{~V}$ at a scan rate of $10 \mathrm{mV} \mathrm{s}^{-1}$. In this way, the partially blocked Pt disk electrode was prepared. After that, the solution was saturated with $\mathrm{H}_{2}$ and the HOR measurement with the electrode was carried out. With this electrode, the upper vertex potential for the HOR measurement was set at $0.4 \mathrm{~V}$ so as not to oxidize the remaining $\mathrm{CO}$. After the HOR measurement, the remaining $\mathrm{CO}$ was quantified by $\mathrm{CO}$ stripping voltammetry. $\mathrm{CO}$ stripping voltammetry was performed in $\mathrm{N}_{2}$ saturated solution and the potential was cycled between 0.02 and $1.2 \mathrm{~V}$ at a scan rate of $10 \mathrm{mV} \mathrm{s}^{-1}$ for 3 times.

\subsection{Observation of Pt disk surface}

The crystallographic orientation of a Pt disk surface was investigated by electron backscatter diffraction (EBSD). The EBSD measurements were conducted using a fieldemission scanning electron microscope (FE-SEM, ULTRA55; Carl Zeiss) operated at an accelerating voltage of $15 \mathrm{kV}$, and the collected EBSD patterns were analyzed using Channel 5 system and a Nordlys II detector (Oxford/HKL). The measurements were carried out at a step size of $0.1 \mu \mathrm{m}$. To fit the sample stage of the FE-SEM, a detachable Pt disk insert (AFED050P040PT, PINE instruments) was used for the analysis. 


\section{Results and Discussion}

\subsection{Change in Koutecky-Levich slope}

Cyclic voltammograms of the Pt disks are shown in Fig. 1. The shapes were very similar to those of the literature [16, 17], thereby the whole system was thought to have been cleaned well. The roughness factors of the Pt disks were determined from these voltammograms to be ca. 1.5 (diameter: $5 \mathrm{~mm}$ ) and ca. 1.6 (diameter: $2 \mathrm{~mm}$ ). The hydrodynamic voltammograms obtained with the Pt disk (diameter: $5 \mathrm{~mm}$ ) for the HOR are shown in Fig. 2. The current density increased with potential and reached a limiting current density below $0.2 \mathrm{~V}$ and looked independent of potential up to about $0.8 \mathrm{~V}$. Then, the current density started to decrease and kept decreasing to the maximum potential. A similar trend was reported in the literature for both aqueous electrochemistry $[18,19]$ and solid-state electrochemistry utilizing a solid polymer electrolyte $[14,20]$. In our past work [10], it was verified that the loss of the HOR current in the high potential region is not caused by the suppression of the inherent Pt activity for HOR of individual sites but caused by site blocking. Therefore, the influence of the site blocking, which relates to effective surface area, $S^{\text {eff }}$, might be obtainable by investigating the relation between mass transport, kinetic current, and potential.

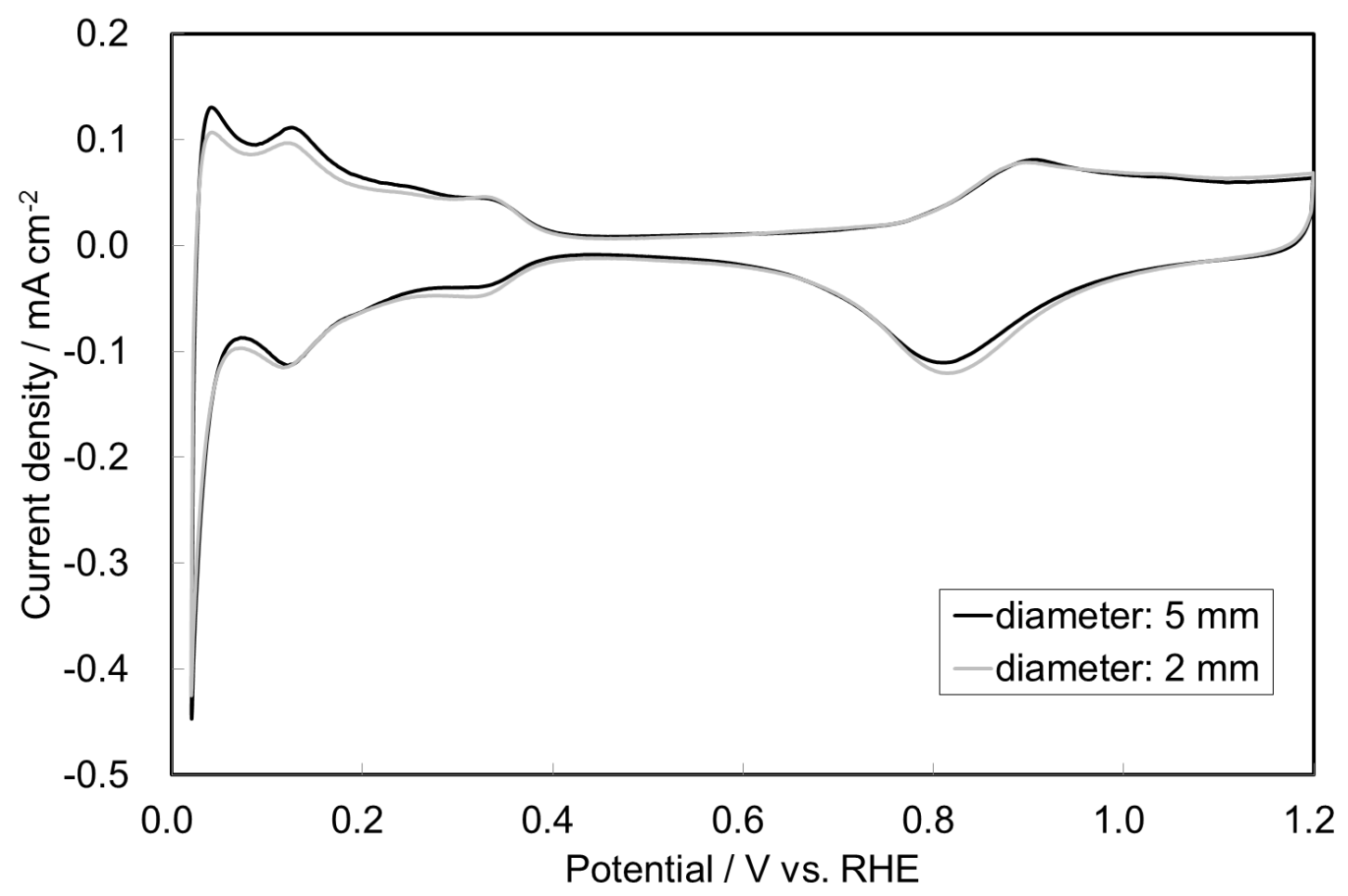

Fig. 1. Cyclic voltammogramms of Pt disk electrodes (diameters: 5 or $2 \mathrm{~mm}$ ) in $0.1 \mathrm{M}$ $\mathrm{HClO}_{4}$ At room temperature. Scan rate: $50 \mathrm{mV} \mathrm{s}^{-1}$. 


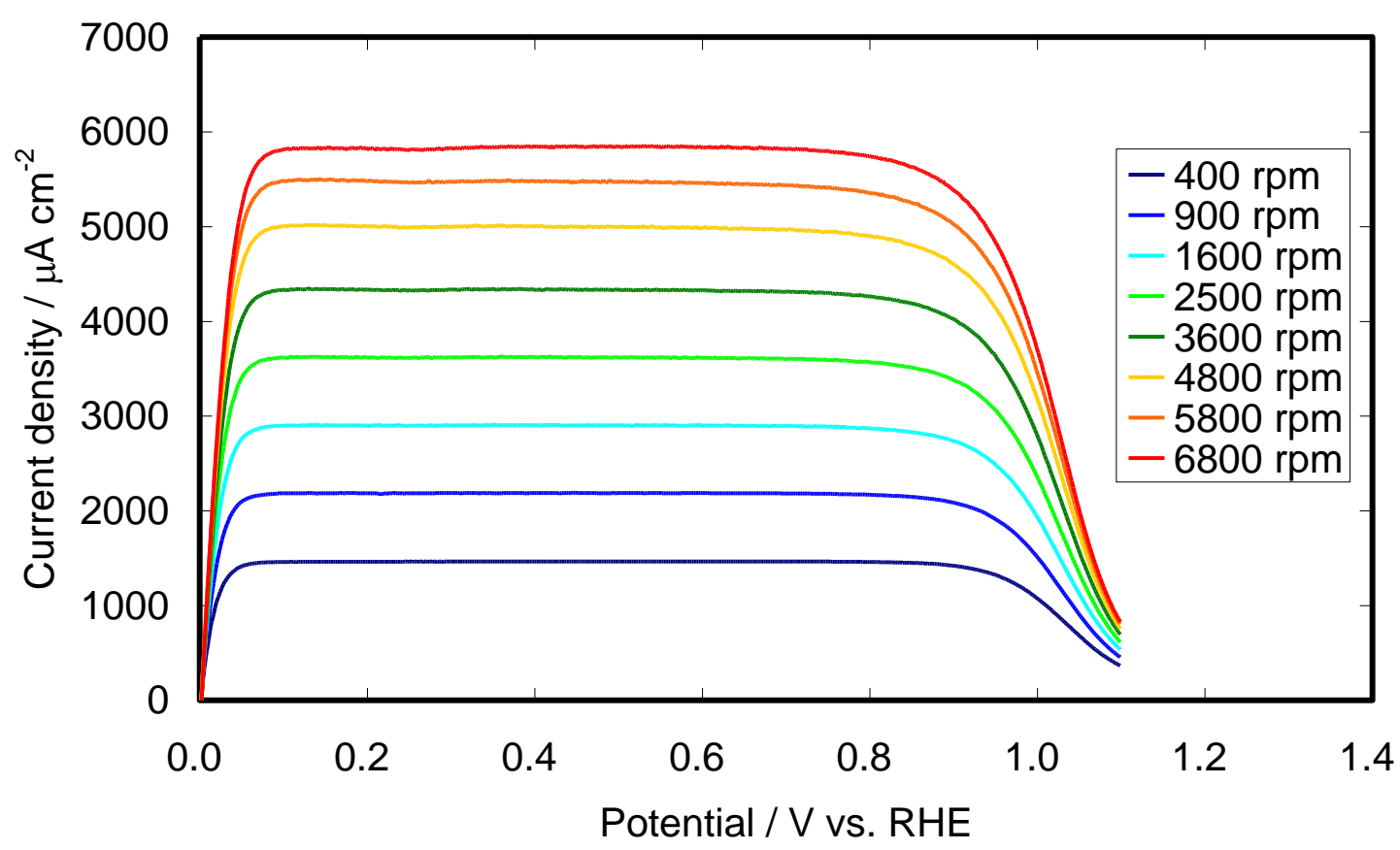

Fig. 2. Hydrodynamic voltammograms over the potential range 0 to $1.1 \mathrm{~V}$ for the HOR on a $5 \mathrm{~mm}$ diameter Pt disk under different rotation rates in hydrogen saturated $0.1 \mathrm{M}$ $\mathrm{HClO}_{4}$ at room temperature. Scan rate: $10 \mathrm{mV} \mathrm{s}^{-1}$

To see the change of the mass transport, Koutecky-Levich (K-L) plots (Fig. 3) were created from the hydrodynamic voltammograms in Fig. 2. Before creating Fig. 3, the background voltammogram obtained in the $\mathrm{N}_{2}$ saturated solution with no rotation was subtracted from each of the voltammograms in order to cancel the influence of currents other than the HOR current (these currents were small and accounted for no more than $3 \%$ of the total current at the maximum potential). The K-L slope changes with rotating speed at high potentials and this change becomes more noticeable as the potential increased. According to the K-L equation (Eq. (2)), the current is expressed as

$$
\frac{1}{i}=\frac{1}{0.62 n F A C_{0} D^{2 / 3} v^{-1 / 6}} \omega^{-1 / 2}+\frac{1}{i_{k}} \quad \text { (2) [21], }
$$

where $A$ is the geometrical electrode area, $C_{0}$ is the concentration of reactant in the bulk, $D$ is the diffusion coefficient of the reactant, $v$ is the kinematic viscosity of the electrolyte, $\omega$ is the angular rotation speed of the electrode and $i_{k}$ is the kinetic current. The K-L slope is determined by the first term of the equation. $C_{0}, D$ and $v$ are the inherent properties of the electrolyte. In terms of the HOR, $n$ is 2 and unlikely to vary. The slope at $1.05 \mathrm{~V}$ was more than twice the one at the limiting current, and therefore only changes in $A$ or $i_{k}$ can accommodate this change in slope (or that the K-L equation does not adequately represent reality). $A$ represents the geometrical area of the disk but it might be related to $S^{e f f}$ by $S^{e f f}=A_{\omega} / A_{g e o}$, where the parameters represent the active area for a given rotation rate divided by the geometric area of the electrode. Alternatively, $i_{k}$ might change with rotation speed. At high rotating rates, hydrogen concentration could have increased at the Pt surface and chemically reduced all of the Pt oxides, resulting in a higher apparent current density (i.e. higher $S^{\text {eff }}$ ) although in this case the slope should be 
constant (case A: the broken line in Fig. 3). Alternatively, the K-L slope depends on $S^{\text {eff }}$ but for some reason, the apparent current density increased at low rotating rates (case B: the dotted line in Fig. 3).

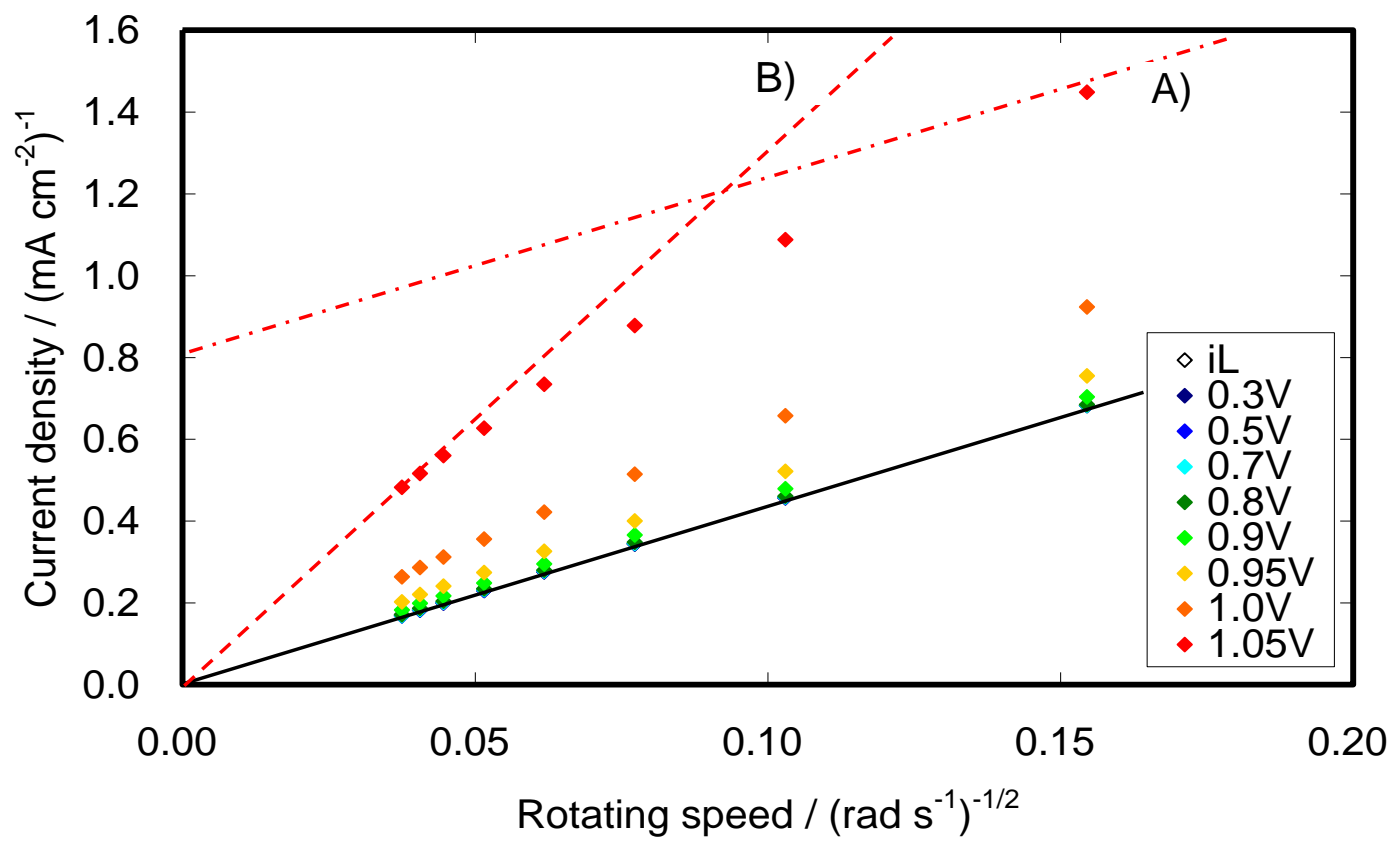

Fig. 3. Koutcky-Levich plots for the HOR on a $5 \mathrm{~mm}$ diameter Pt disk in hydrogen saturated $0.1 \mathrm{M} \mathrm{HClO}_{4}$ at room temperature. (Case A; the slope is constant even at 1.05 $\mathrm{V}$, case $\mathrm{B}$; the intercept is constant)

In order to understand the meaning of the change of the K-L slope, applying a different adsorbate which is not easily removed, even under a high rotating speed, was thought to be helpful. $\mathrm{CO}$ was used as the alternative adsorbate, as it is well known that $\mathrm{CO}$ specifically adsorbs onto $\mathrm{Pt}$ and cannot be easily reduced by hydrogen. Partial $\mathrm{CO}$ blocking was done according to the procedure described in 2.4. After partial blocking with $\mathrm{CO}$, the hydrodynamic voltammograms were obtained under different rotation rates (Fig. 4). Comparing the voltammograms in Fig. 2 and 4, it was found that the limiting current decreased with the partial blocking. This indicates that even with the same geometrical area, the limiting current can vary probably depending on $S^{\text {eff }}$. Fig. 5 shows the comparison of K-L plots obtained with and without the partial blocking. With the partial blocking, the limiting current at $0.3 \mathrm{~V}$ clearly decreased compared to that without the partial blocking and its slope looked similar to the one obtained at a high potential $(0.95 \mathrm{~V})$ without the partial blocking. This result suggested that the change in K-L slope is caused by the decrease in $S^{\text {eff }}$, and $i_{k}$ for HOR is high enough regardless of the potential and coverage. The idea that $i_{k}$ did not matter to the current even at a high potential was explained in our past work [10] as well. However, the relation between $S^{\text {eff }}$ and the K-L slope needs to be clarified more for quantitative understanding. 


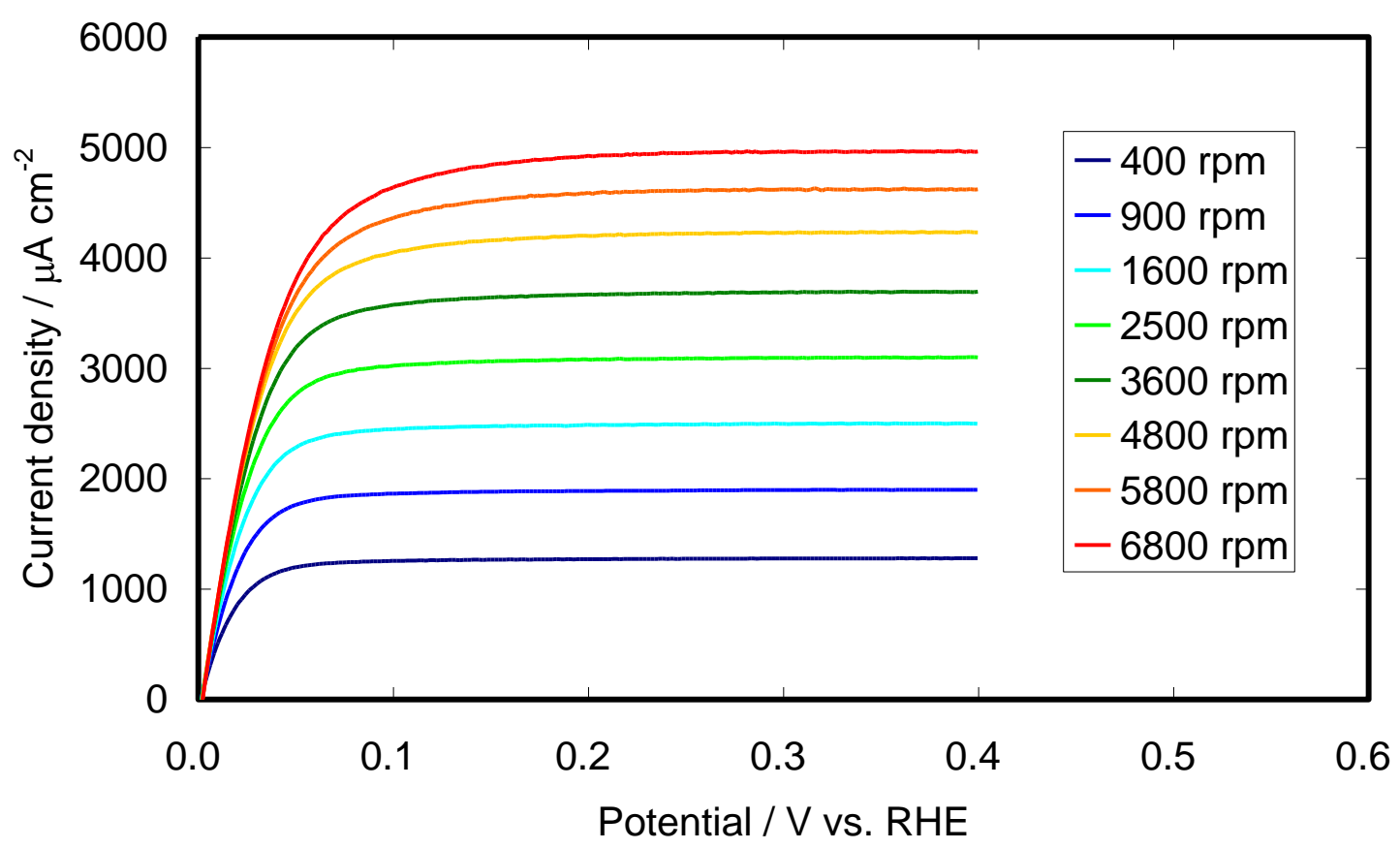

Fig. 4. Hydrodynamic voltammograms over the potential range 0 to $0.4 \mathrm{~V}$ for the HOR on a $5 \mathrm{~mm}$ diameter Pt disk (after partial CO blocking) under different rotation rates in hydrogen saturated $0.1 \mathrm{M} \mathrm{HClO}_{4}$ at room temperature. Scan rate: $10 \mathrm{mV} \mathrm{s}^{-1}$

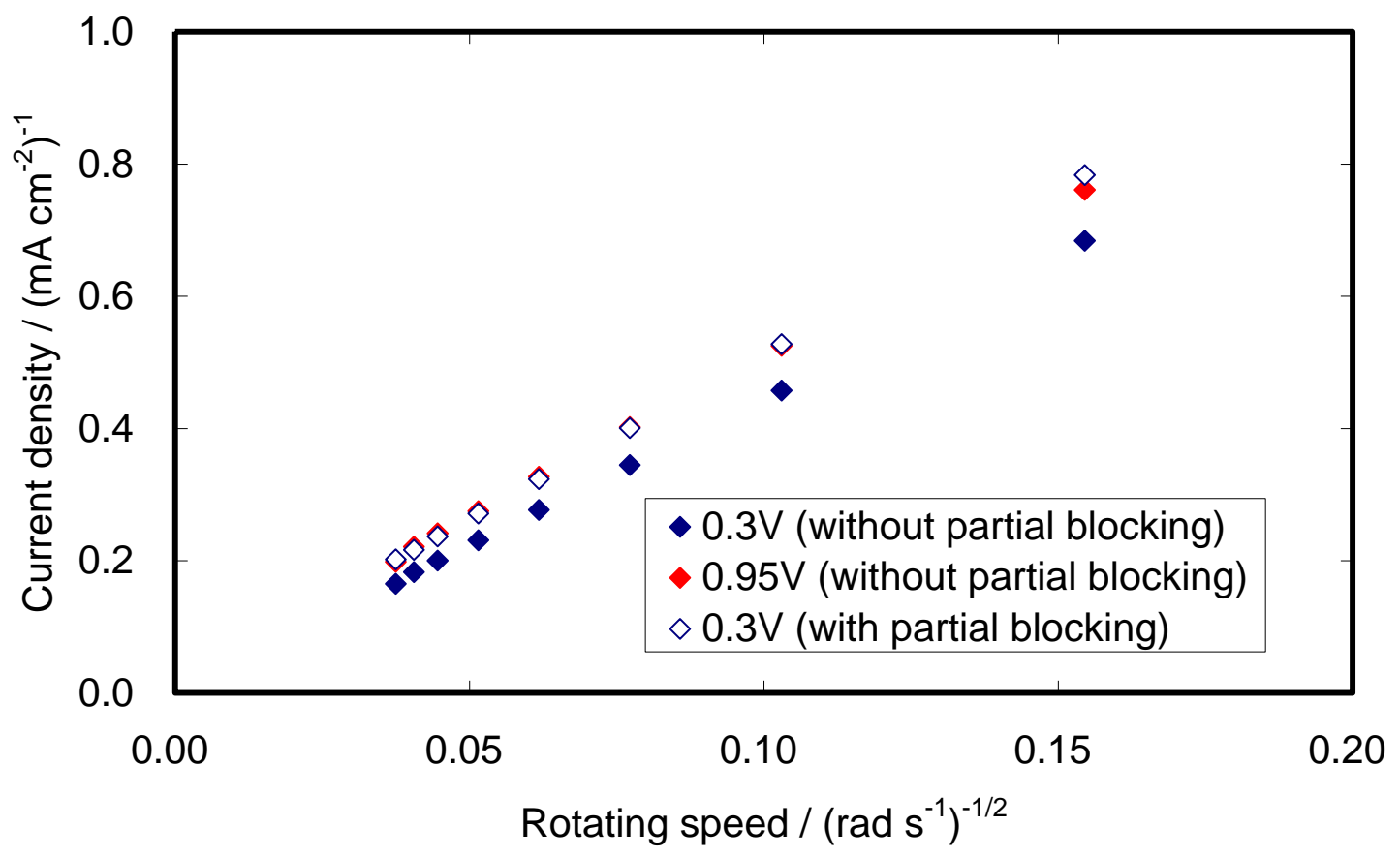

Fig. 5. Koutcky-Levich plots for HOR at Pt disk electrode (with and without partial CO blocking) at room temperature. 


\subsection{Numerical approaches for understanding the change in $K-L$ slope}

The rotating disk systems involves a complex interplay between diffusion of reactants through a boundary layer and subsequent reaction of those reactants at the electrode surface. The typical boundary layer thickness during the RDE studies we have performed are in the range 13-4 $\mu \mathrm{m}$ (correspond to $400-4000 \mathrm{rpm}$ ). It is typically implicitly assumed that the blocking species is uniformly distributed across the surface with an average separation much smaller than the boundary layer thickness, so that the electrode surface may be treated as being homogeneous. This may not be the case for two distinct reasons. The first is that the electrode is polycrystalline and is composed of sets of crystals of specific size and orientation. Fig. 6 shows an inverse pole figure map of the Pt disk electrode in the vertical direction. The figure shows that crystallographic orientations were random. Each crystal facet on the surface has a slightly different electrochemical response and will, for example, have different oxide coverages at the same electrochemical potential. The second is that adsorbates may show some lateral interaction leading to repulsive or attractive behavior, as typically modeled using the Frumkin isotherm. In the case where such lateral interactions are attractive, clustering of the adsorbates (be it oxide or carbon monoxide) will lead to islands of the adsorbate [22, 23].This means that at the microscopic level, the coverage is not homogeneous, but shows patches where the coverage is high, and other areas where the coverage is low. Where the domain size of those inactive regions is similar or greater than the boundary layer thickness, then we might expect significant deviation from the standard KLformalism. Gas concentration profiles around the partially blocked electrodes with different sizes of inactive domain and different boundary layer thicknesses were calculated (Fig. 7) based on a diffusion model (detail will be discussed in the next section). In our simulation approach, we seek to determine whether the size and distribution of these nano domains can influence the K-L response as we see above.
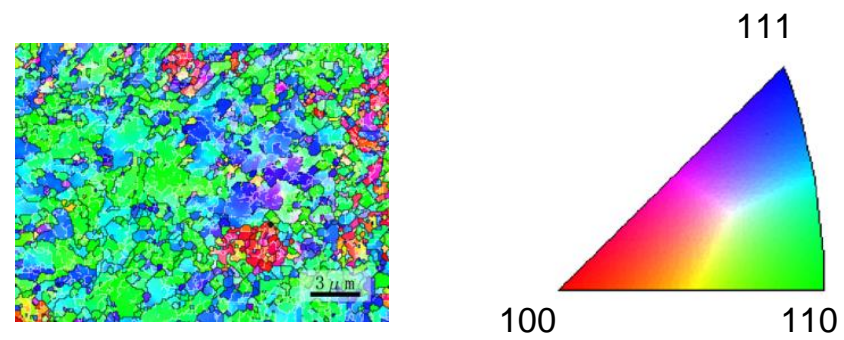

Fig. 6. An inverse pole figure map of the Pt disk electrode showing crystallographic orientations in the vertical direction. $15 \mathrm{kV}$ acceleration voltage and $0.1 \mu \mathrm{m}$ step size. 

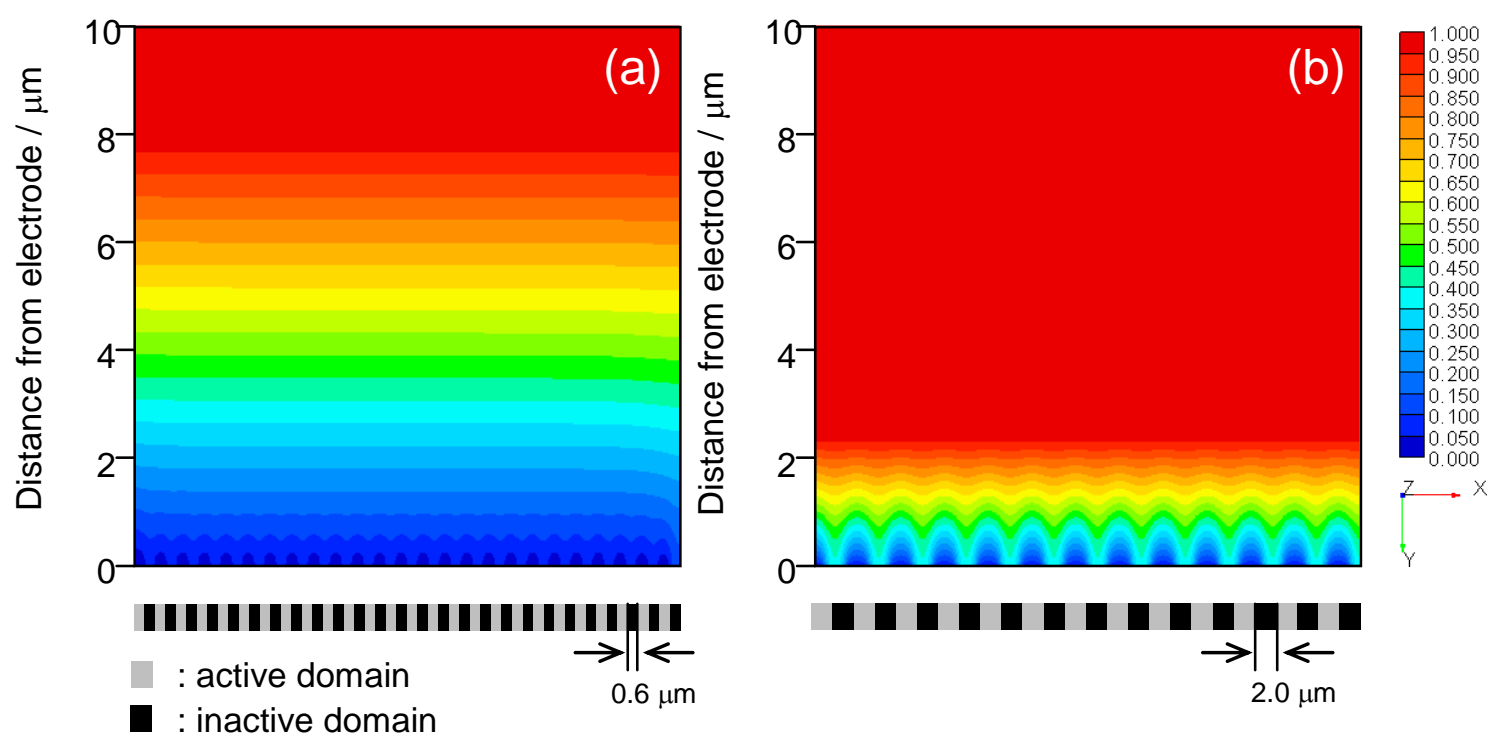

Fig. 7. Gas concentration profiles above the partially blocked electrodes. (a) simulation in which the boundary layer thickness is $8.0 \mu \mathrm{m}$ with a $0.6 \mu \mathrm{m}$ inactive domain size; and (b) simulation in which the boundary layer thickness is $2.5 \mu \mathrm{m}$ with a $2.0 \mu \mathrm{m}$ inactive domain size.

\subsubsection{Diffusion-only approximation}

Strmcnik et al. investigated the relation between the surface coverage and the rate of electrocatalytic reactions [24]. They simulated the current-time response for HOR with $10 \%$ active surface area and $100 \mathrm{~nm}$ sized active domains. As was done in their work, numerical approaches were thought to be useful to understand the relation between $S^{\text {eff }}$ and the K-L slope. However, in this case we intend to study the steady state current under RDE conditions. First, a simple diffusion model was applied. When the mass transport phenomenon in the boundary layer is diffusion dominant, the transport problem can be solved with a diffusion model. The model used was based on the 2-dimensional diffusion equation below.

$$
\frac{\partial C}{\partial t}=D\left(\frac{\partial^{2} C}{\partial x^{2}}+\frac{\partial^{2} C}{\partial y^{2}}\right)
$$

Outside the diffusion dominated region, the solution is assumed to be well-stirred and at constant reactant concentration. The dimension of the model was fixed as $50 \mu \mathrm{m}$ in $x$ direction (Pt disk plane direction) and $10 \mu \mathrm{m}$ in $y$ direction (boundary layer direction). In each direction, the segment size was fixed as $0.1 \mu \mathrm{m}$, resulting in 500 and 100 segments in $x$ and $y$ directions, respectively. $\theta$ and the inactive domain size, $l$ were varied for a parametric study and the calculation was done with different boundary layer thickness to obtain K-L plots. The thicknesses of the boundary layer applied to the calculation are designated in Table 2.

Dispersion patterns of the inactive domains can vary even with the same $\theta$ and $l$. Therefore, several different patterns were prepared and the calculation was repeated for each pattern and then the results were averaged. An example of the dispersion patterns are shown in Fig. 8. Inactive regions were treated as a reflective wall. On the other hand, hydrogen concentration at the active surface was regarded as 0 because the limiting current should be observed there. The current was obtained from the gradient of the 
hydrogen concentration at the surface. The hydrogen concentration at each segment was calculated using Eq. (3) and repeated until the deviation in the current in the whole system between time steps became lower than $0.01 \%$ to simulate a steady state. The time step, $\Delta t$, and $D$ are also shown in Table 2 even though they do not affect the result as the concentration profile at steady state will not change with those parameters. In Table 2, the boundary layer thicknesses, $\delta$, were estimated using Eq. (4) and shown with rotation speeds.

$\delta=1.61 D^{1 / 3} \omega^{-1 / 2} v^{1 / 6} \quad$ (4) [21].

$D$ was determined to be the double of the oxygen diffusivity in $0.1 \mathrm{M} \mathrm{HClO}_{4}\left(1.93 \times 10^{-}\right.$ ${ }^{5} \mathrm{~cm}^{2} \mathrm{~s}^{-1}$ for oxygen) [25] assuming that the ratio of the diffusivities for hydrogen and oxygen is $2: 1$. $\Delta t$ was determined by the following equation (Eq. (5)) so as to secure the calculation stability.

\section{$\frac{D \Delta t}{d x d y} \leq \frac{1}{4} \quad(5)$}

where $d x$ and $d y$ are the segment sizes in each direction.

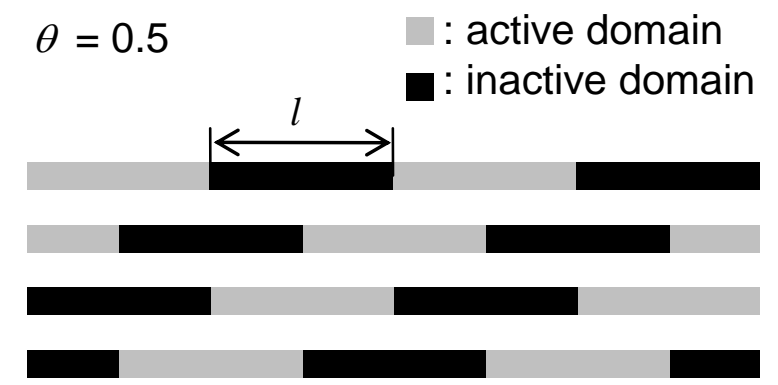

Fig. 8. Examples of different dispersion patterns of inactive domain on Pt surface $(\theta=$ $0.5)$.

Table 2

Parameters for diffusion model

Boundary layer thickness $\delta / \mu \mathrm{m}$ (rotation rate / $\mathrm{rpm})$

Coverage, $\theta /$ -

2.5 (10273), 3.0 (7134), 4.0 (4013), 6.0 (1783), 8.0 (1003)

Inactive domain size, $l / \mu \mathrm{m}$

Diffusion coefficient, $D / \mathrm{cm}^{2} \mathrm{~s}^{-1}$

$0.5,0.75$

Time step, $\Delta t / \mathrm{s}$

$0.6,2.0$ (only for $\theta=0.5$ )

$3.86 \times 10^{-5} *$

*Ref. 25.

Calculated K-L plots based on the diffusion model are shown in Fig. 9. The solid line in Fig. 9 was obtained from the limiting current density in Fig. 3. The other lines were obtained from the calculated ineffectiveness factor, $\chi^{\text {ineff }}$, and the solid line in Fig. 9. $\chi^{\text {ineff }}$ was calculated from Eq. (6) below.

$$
\chi^{\text {ineff }}=1-\frac{I}{I_{\text {bare }}}
$$

where, $I_{\text {bare }}$ and $I$ are the currents with no active patches (i.e. the surface is uniformly active) and those with active and inactive domains, respectively. As can be seen in Fig. 9, the plots only shifted vertically regardless of the parameters, and there was no change in the gradient. This change was similar to the case when a polymer film, such as Nafion ${ }^{\circledR}$ film was formed onto a $\operatorname{RDE}[15,26]$. There was no change in the K-L slope although, of 
course, the intercept shifted. A similar trend was observed by Nosek et al. [27] in their study of mass transport though a colloidal particle monolayer on a gold RDE. Their model was also based on diffusion. These trends do not correspond to those shown in experimental plots (Fig. 3).

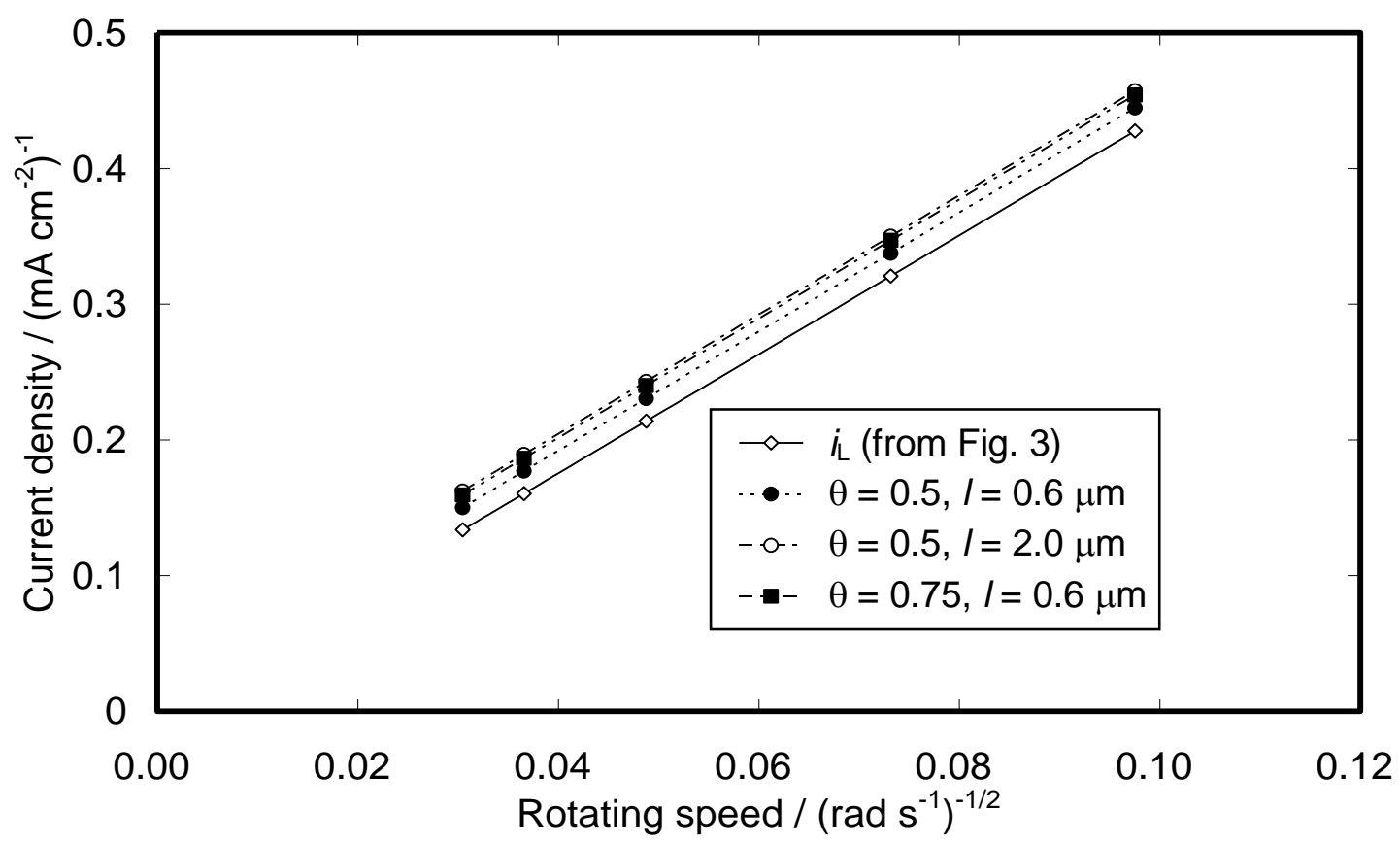

Fig. 9. Calculated Koutecky-Levich plots based on the diffusion model with different parameters.

\subsubsection{Convection-only approximation}

In order to test whether the failure to replicate the experimental results was due to the assumption of pure diffusion, a convection model was also examined. Solution of the convection-diffusion equation for a Rotating Ring-Disk Electrode, RRDE, provides a relation between the ring current and the disk current expressed as:

$$
i_{R}=i_{D} \frac{\left(r_{3}{ }^{3}-r_{2}{ }^{3}\right)^{2 / 3}}{r_{1}{ }^{2}} \quad \text { (7) [21], }
$$

where $i_{R}$ and $i_{D}$ are the ring current and disk current, respectively, $r_{1}$ is the radius of the disk, $r_{2}$ is the inner radius of the ring and $r_{3}$ is the outer radius of the ring. $i_{R}$ is determined just by the geometry of the electrode. We can use this model to estimate the effectiveness factor by considering the case when the gap between the ring and the disk, $\Delta=r_{2}-r_{1}$, is much larger than the boundary layer thickness so that the influence of diffusion is negligible. Like the diffusion model, the geometry of the electrode can vary even with the same $\theta$. For this reason, it is necessary to investigate the influence of the geometry.

In the case of the RRDE, $\theta$ can be expressed as

$$
\theta=\frac{\pi\left(r_{2}^{2}-r_{1}^{2}\right)}{\pi r_{3}^{2}}
$$


In Eq. (8), $r_{2}$ can be replaced by $\Delta+r_{1}$. Therefore, the relation between $r_{1}, r_{2}$ and arbitrarily determined $r_{3}$ can be determined by $\Delta$ for a certain value of $\theta$. With this relation, $i_{R}$ and $i_{D}$ are obtained. $\chi^{\text {ineff }}$ of the electrode was calculated as well. In this case, $I_{\text {bare }}$ in Eq. (6) would be the disk current for the bare electrode with radius $r_{3}$. The relation between the electrode geometry and the apparent ineffectiveness, which is expressed as $\chi^{\text {ineff }} / \theta$, is shown in Fig. 10. As shown in Fig. 10, the apparent ineffectiveness factor varied with the geometry of the electrode. When $r_{1} / r_{3}$ is 0 , in other words, the disk radius is 0 , the geometry of the electrode should only be a ring electrode. On the other hand, when the apparent ineffectiveness is 1 , namely when $\chi^{\text {ineff }}$ is the same as $\theta$, the electrode is a disk electrode. In other cases, the electrode should have a ring and a disk. As it was thought that the inactive domain disperses homogeneously, averaged values seemed appropriate for comparison. However, Fig. 10 is just an example assuming a simple RRDE, therefore, $\chi^{\text {ineff }}$ was calculated with the same dispersion patterns as in the case of the diffusion model above.

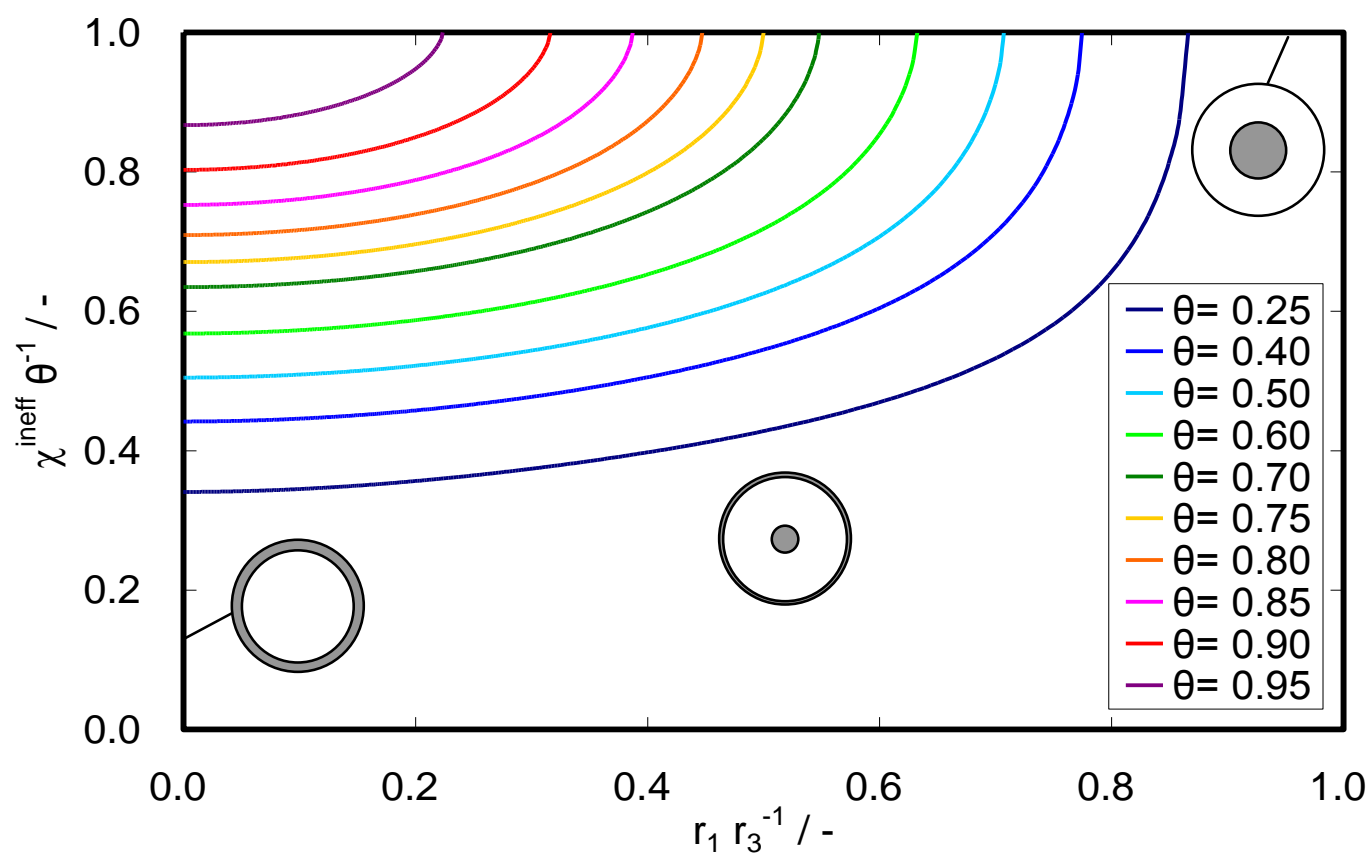

Fig. 10. A relationship between the geometry of the electrode and apparent ineffectiveness factor based on the convection model.

As the radius of the electrode was seen to affect the apparent ineffectiveness, the model size was expanded to the actual radius of the Pt disk $(2.5 \mathrm{~mm})$ from the one for the diffusion model $(50 \mu \mathrm{m})$. Parameters (coverage and inactive domain size) for the convection model were the same as those of the diffusion model. To make the difference clearer, $\theta=0.9$ was also applied with $l=9.0 \mu \mathrm{m}$. Calculated K-L plots based on the convection model are shown in Fig. 11. As shown in Fig. 11, the effectiveness also varies with the geometry. However, contrary to the diffusion model, there was no change in the intercept and the gradient became greater with increasing of $\theta$ and $l$. Although the gradient changed with $\theta$ and $l$, it was independent of the rotating speed and the trend was slightly different from the experimental plots. When the rotating speed decreased, the 
K-L slope of the experimental plots got closer to the observed gradient of the limiting current (the chained line in Fig. 11). When the limiting current is observed (on a bare RDE surface), mass transport in the boundary layer is usually treated as diffusion [21]. Moreover, the influence of convection should be weakened when the rotating speed becomes lower. From these points, the change in the K-L slope was thought to express the transition of mass transport phenomenon, namely, from diffusion dominant in the lower rotating speed region to convection dominant in the higher rotating speed region. This suggested that a full convective-diffusion model is required to reproduce the trend in the experimental plots.

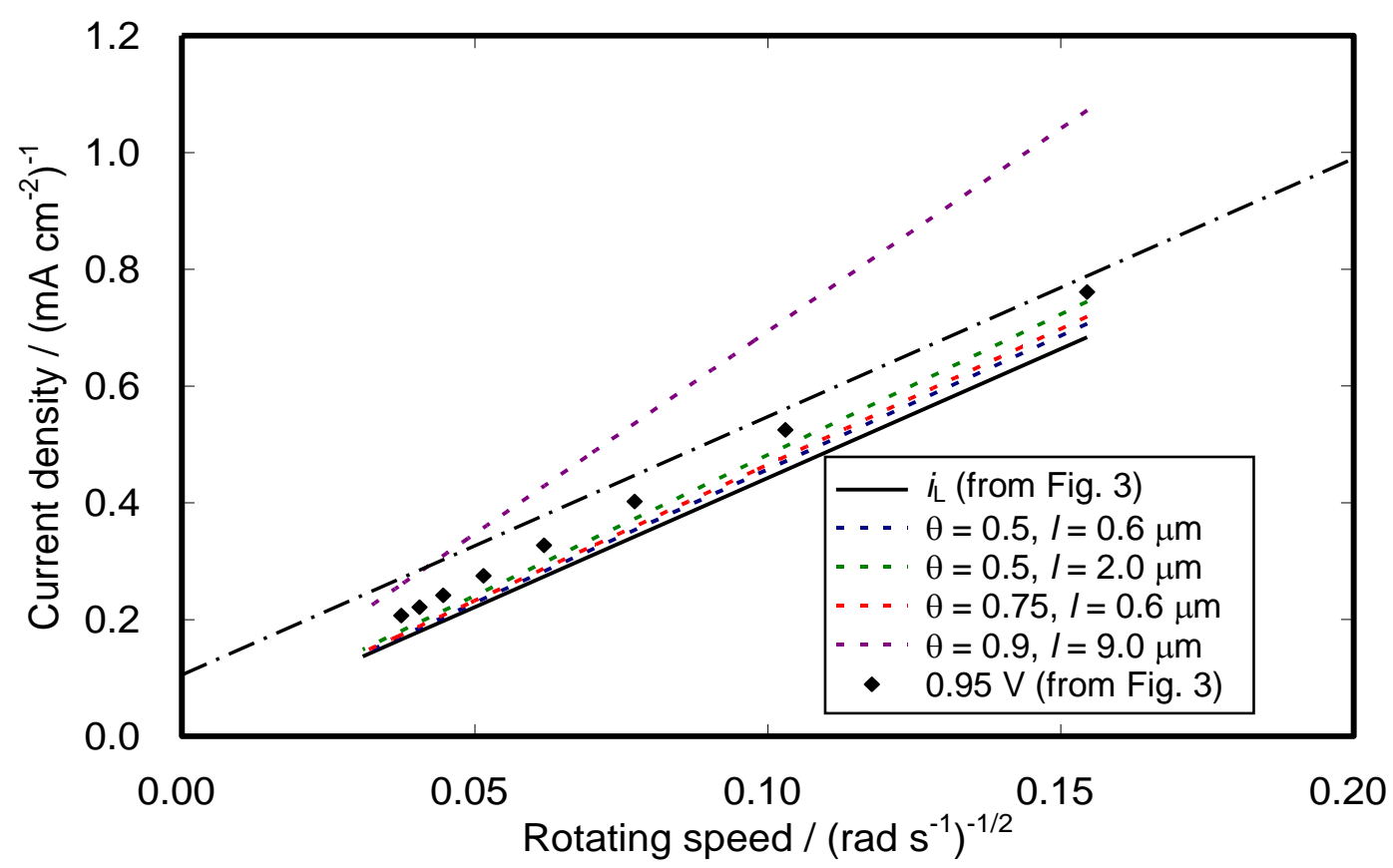

Fig. 11. Calculated Koutecky-Levich plots based on the convection model with different $\theta$ and inactive domain size. (Chained line; a drawing having the same gradient as the limiting current.)

\subsubsection{Full Convection-diffusion model}

From the results obtained in 3.2.1 and 3.2.2 it is clear that a pure diffusion model will only replicate the vertical shift of the K-L line (i.e. decrease in apparent $i_{k}$ ) and that a pure convection model will predict a change in slope, especially at low disk rotation rates. Hence, it is necessary to apply a full convection-diffusion model. The model used is similar to that of the diffusion model. The thickness of the boundary layer was increased to $20 \mu \mathrm{m}$ so that the validity of the calculation could be secured even with low rotation rates. The equation was replaced by the convective-diffusion equation,

$v_{x}\left(\frac{\partial C}{\partial x}\right)+\frac{v_{\phi}}{x}\left(\frac{\partial C}{\partial \phi}\right)+v_{y}\left(\frac{\partial C}{\partial y}\right)=D\left[\frac{\partial^{2} C}{\partial x^{2}}+\frac{\partial^{2} C}{\partial y^{2}}+\frac{1}{x} \frac{\partial C}{\partial x}+\frac{1}{x^{2}}\left(\frac{\partial^{2} C}{\partial \phi^{2}}\right)\right]$

where $\phi$ is the dimension in the rotation direction and $v$ is the velocity profile of the solution in each direction. $v_{x}$ and $v_{y}$ were already solved by Levich [28] and shown in the literature [21]. Assuming the symmetry in $\phi$ direction, Eq. (9) can be simplified by 
eliminating the terms that include $\phi$. Instead of the thickness of the boundary layer, the rotation rate was varied. The boundary conditions, the dispersion patterns of inactive domains and the termination condition were the same as those of the diffusion model. The parameters used in the convective-diffusion model are summarized in Table 3 . The kinematic viscosity of $0.1 \mathrm{M} \mathrm{HClO}_{4}$ was taken from Ref. 29.

Table 3

Parameters for convective-diffusion model

\begin{tabular}{lc}
\hline Rotating speed $/ \mathrm{rpm}$ & $400(12.7), 1000(8.0), 2000(5.7), 4000(4.0), 7000(3.0), 10000(2.5)$ \\
(Boundary layer thickness $\delta / \mu \mathrm{m})$ & 0.5 \\
Coverage, $\theta /$ - & $1.0,2.0,4.0,6.0,8.0$ \\
Inactive domain size, $l / \mu \mathrm{m}$ & $1.009 \times 10^{-2 * 1}$ \\
Kinematic viscosity $/ \mathrm{cm}^{2} \mathrm{~s}^{-1}$ & $3.86 \times 10^{-5} * 2$ \\
Diffusion coefficient, $D / \mathrm{cm}^{2} \mathrm{~s}^{-1}$ & $6.47 \times 10^{-7}$ \\
Time step, $\Delta t / \mathrm{s}$ &
\end{tabular}

*1: Ref. 29, *2: Ref. 25

Calculated K-L plots based on the convective-diffusion model are shown in Fig. 12. As can be seen in Fig. 12, the calculated curves show the change in the gradients with rotating speed. They became greater with increasing of rotation speed. With larger inactive domain size, the gradient starts to increase from the lower rotating speed. As explained above, when the boundary layer thickness becomes much thinner than the size of the insulator of the RRDE, lateral diffusion would become negligible. Likewise, mass transport should become diffusion dominant with thicker boundary layers, in other words, at lower rotation speeds, even with the largest inactive domain size. The calculation showed this trend well. With these 3 different models, it is seen that the change in K-L slope expresses the transition of mass transport phenomenon. Although a Pt disk was applied to this study to simplify the analysis, the models might be applicable to more practical cases which utilize relevant catalysts such as $\mathrm{Pt}$ nano particles and $\mathrm{Pt} / \mathrm{C}$ catalysts. However, forming a very low loading catalyst layer is still necessary so as not to complicate the system. For instance, the minimum practical loading achievable on an RDE utilizing a $60 \mathrm{wt} . \% \mathrm{Pt}$ on carbon catalyst is about $10 \mu \mathrm{g}_{\mathrm{Pt}} \mathrm{cm}^{-2}$. This layer is composed of $\sim 600 \mathrm{~nm}$ carbon agglomerates to a depth of 2-3 $\mu \mathrm{m}$. Hence the layer thickness is about the same as the boundary layer thickness, and significant mass transport effects might be expected in the y-direction. Zalitis et al. succeeded in producing ultra thin, uniform catalyst layers by the vacuum filtered catalyst method [14]. Such catalyst layers seem applicable to the models. 


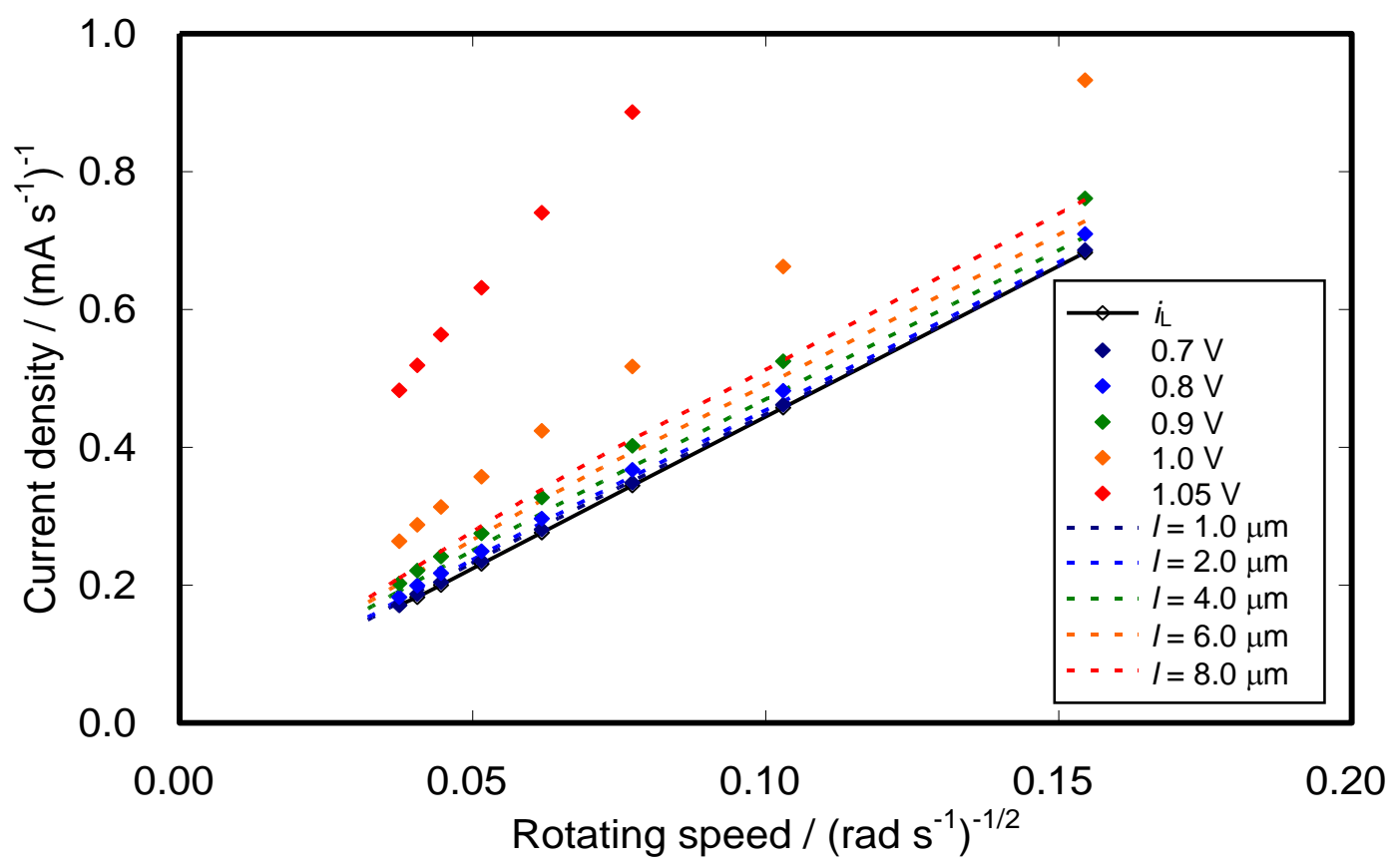

Fig. 12. A comparison of the experimental Koutecky-Levich plots (in Fig. 3) with calculated ones based on the convective-diffusion model with different inactive domain sizes and $\theta$ of 0.5 .

\subsection{Quantitative analysis of coverage and inactive domain size}

As explained above, when the rotating speed is extremely high, the transport phenomenon would be convection dominated. In such a case, the effectiveness, which is defined as $I / I_{\text {bare }}$ could be obtained using the convection model. However, the effectiveness depends not only on $\theta$ but on the inactive domain size even with the convection model. The relation among effectiveness, $\theta$ and inactive domain size for the $5 \mathrm{~mm}$ Pt disk was calculated (Fig. 13). This relation also depends on the size of the disk electrode. On the other hand, $\theta$ and inactive domain size are probably independent of the disk size. Therefore, it was thought possible to determine those parameters from the K-L plots with different disk sizes. 


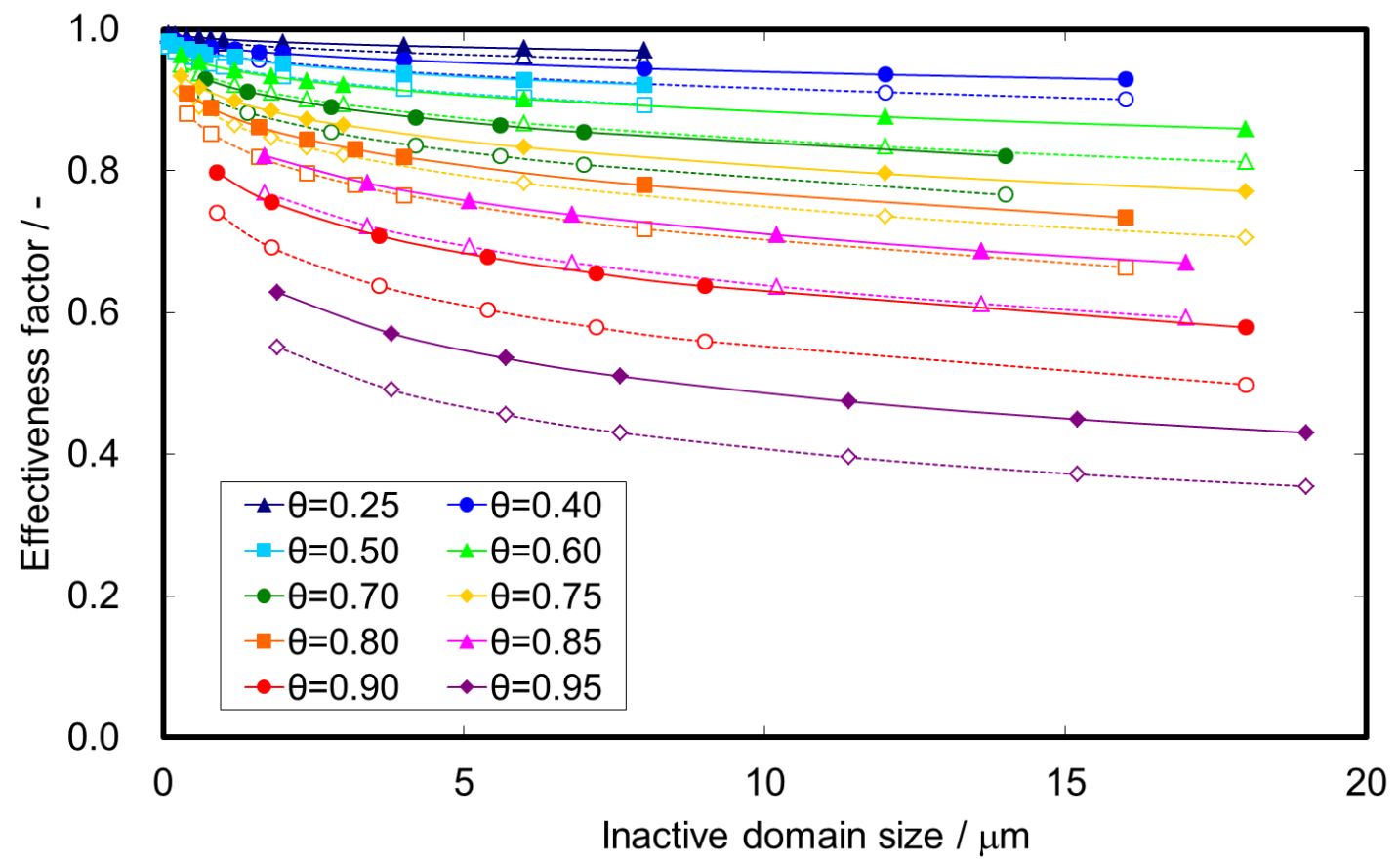

Fig. 13. A relationship among effectiveness factor, $\theta$ and inactive domain size based on the convection model for different sizes of Pt disks (open symbols: $2 \mathrm{~mm}$ diameter, solid symbols: $5 \mathrm{~mm}$ diameter).

The hydrodynamic voltammograms for the $2 \mathrm{~mm}$ Pt disk were obtained in the same way as the $5 \mathrm{~mm}$ Pt disk. K-L plots were then created (Fig. 14). For a further comparison with the result obtained with a MEA [29], K-L plots obtained from the cathodic scan were created. Similar to the case of the larger size of Pt disk, the relation among effectiveness factor, $\theta$ and inactive domain size was also calculated based on the convection model (Fig. 13). A comparison of the two results shows that the trends are the same but the values are different. The smaller Pt disk showed lower effectiveness in any cases. This is probably because the influence of convection becomes stronger where the distance from the center of the rotation is large. The K-L slope at a high rotation rate was evaluated by extrapolation. The range of the rotating rate for the extrapolation was set as $7.0 \times 10^{7} \mathrm{rpm}$ so that $\delta$ would become ca. $1 \mathrm{~nm}$ (according to Eq. (4)), which seemed much smaller than the expected inactive domain size.

In this case, the influence of diffusion becomes negligible. In a real situation, such high rotation are not possible due to cavitation effects in the electrolyte. However, in terms of extrapolation, such limitations should not matter. The ratio of the slope at a certain potential to that at $0.3 \mathrm{~V}$, where the limiting current density was observed, was then calculated. This ratio corresponds to $1-\chi^{\text {ineff }}$ based on the convection model.

From the extrapolation, effectiveness factors with different disk diameters at an extremely high rotation speed were estimated (Fig. 15). Similar to Fig. 13, the smaller Pt disk showed lower effectiveness. This is probably the same reason as for the difference in Fig. 13. From Fig 13 and 15, it seems possible to estimate coverage and inactive domain size because both of them are probably independent of disk diameter. The process of the estimation was as follows. 1) to compare the values of effectiveness in both figures at a certain potential, 2) to find the coverage where the inactive domain size becomes almost the same and 3) to determine the values of inactive domain size from coverage and effectiveness. 


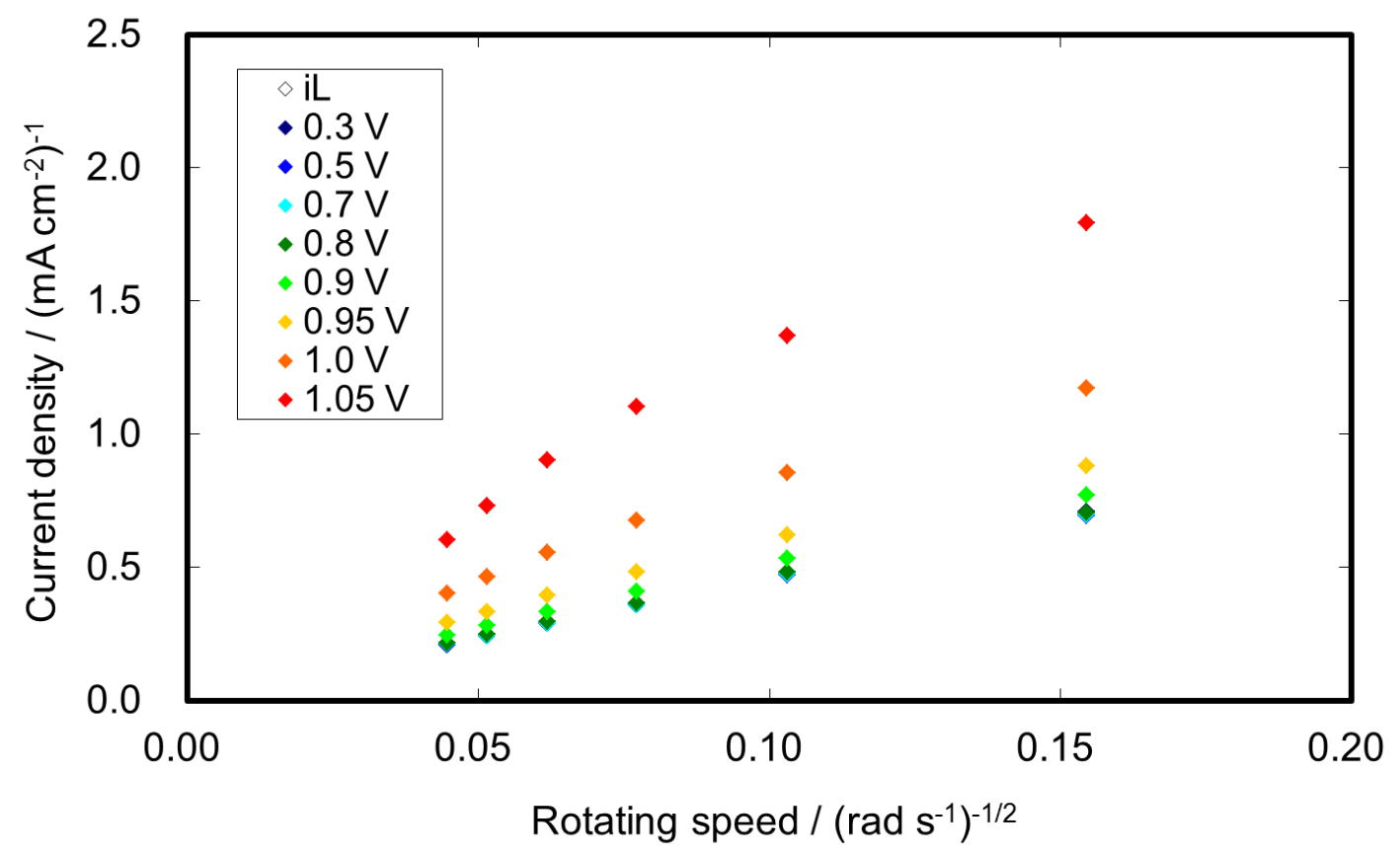

Fig. 14. Koutcky-Levich plots for the HOR on a $2 \mathrm{~mm}$ diameter Pt disk in hydrogen saturated $0.1 \mathrm{M} \mathrm{HClO}_{4}$ at room temperature.

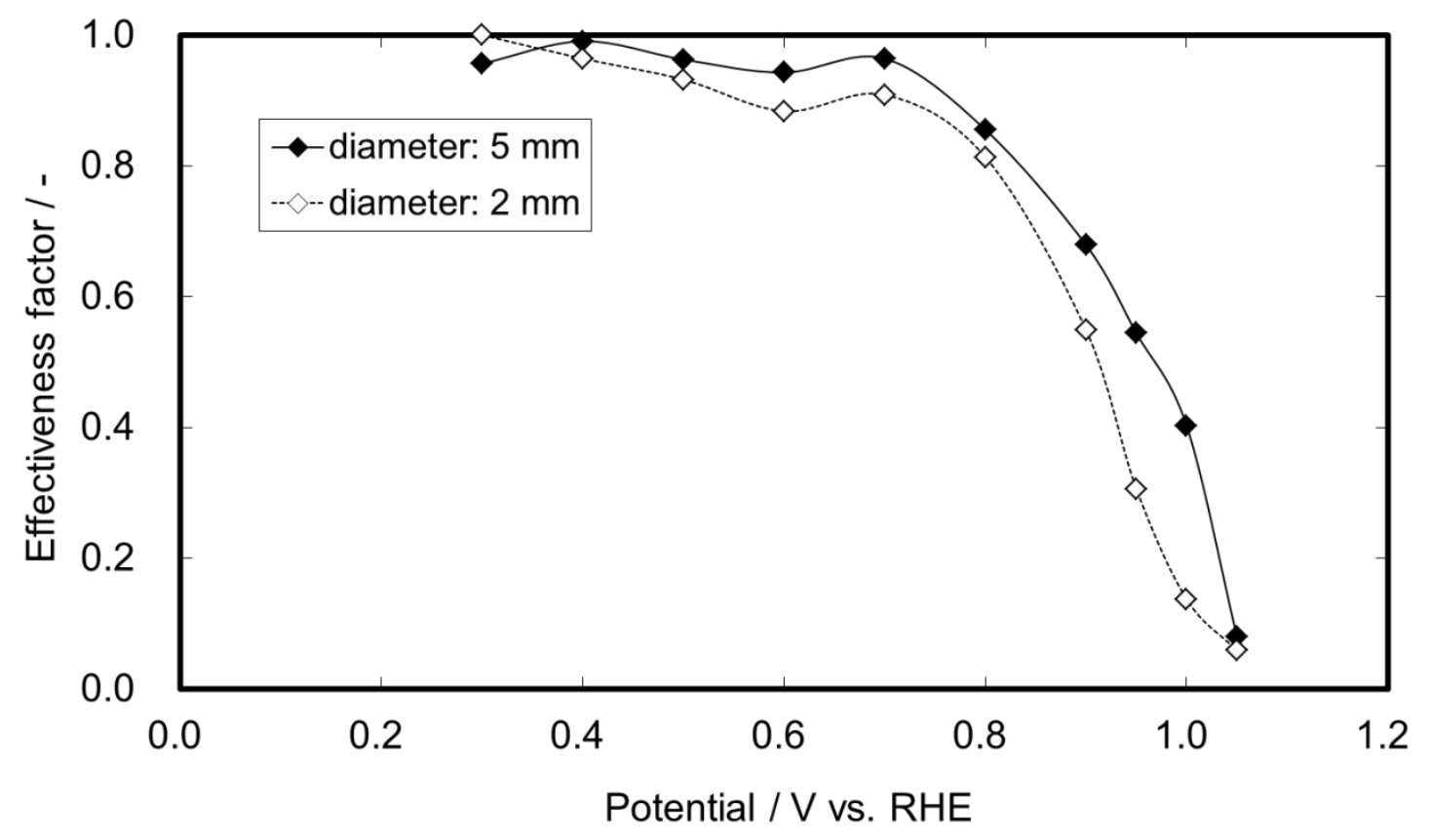

Fig. 15. Estimated effectiveness factors at $7.0 \times 10^{7} \mathrm{rpm}$ as a function of potential with different disk diameters.

The evaluated $\theta$ was compared with the case of the MEA in Fig. 16. As written in our previous work [10], $\chi^{\text {ineff }}$ in the MEA was defined as $1-R_{x V} / R_{0.3 V}$, where $R$ is the hydrogen transport resistance towards the Pt surface. Strictly speaking, $\chi^{\text {ineff }}$ is not exactly equal to $\theta$, however, they do not seem far from each other because the diffusion layer thickness in the MEA, which corresponds to the ionomer thickness, was thought to 
be extremely thin $(2 \sim 3 \mathrm{~nm})$ [30]. Both $\chi^{\text {ineff }}$ and $\theta$ increased with increasing potential. $\theta$ could not be estimated over $0.9 \mathrm{~V}$ and less than $0.4 \mathrm{~V}$ because the calculated effectiveness was too high or low comparing to the model (Fig. 13). Over 0.6 V where it is said that Pt oxide can be formed, the MEA showed higher values than the RDE. The measurement conditions for HOR in the case of the MEA were different from that of the RDE. The conditions are summarized in Table 1. As shown in Table 1, many conditions were different. Among them, the difference in electrode shape might have caused the difference in $\theta$. It was reported that the size of the Pt particle affects Pt oxide formation notably [31]. As the size of the Pt particle in the MEA was very small (about $3 \mathrm{~nm}$ [15]), the growth of $\mathrm{Pt}$ oxide is known to be shifted to lower potential compared to polycrystalline platinum, hence this would give rise to a difference in $\theta$. The difference in electrolytes could have a large influence on $\theta$. Although it was also reported that the Nafion ${ }^{\circledR}$ ionomer [16] can suppress HOR, its dependence on potential has not been clarified yet. Although to investigate the influence of the electrolyte might be difficult with the method using MEAs, it should be much easier with the new method using an RDE. To analyze the difference in $\theta$, further study is necessary. Inactive domain size started to increase notably over $0.7 \mathrm{~V}$. As this trend is similar to that of Pt oxide growth [32], the size might express the size of Pt oxide on the Pt disk electrode.

Although further investigation might be necessary to validate the quantitative accuracy of the analysis when the effectiveness is high enough, the analysis suggests that the effective surface area for electrochemical reaction does decrease notably from relatively low potential, even though it is not obvious in the measured currents due to its being masked by mass transport. The analysis also suggests that there is a large difference in $S^{\text {eff }}$ with different methods, namely, estimation from the amount of $\mathrm{Pt}$ oxides (see Ref. 30) and the analysis derived from mass transport properties. This difference indicates that other causes are dominant at low potential region for the decrease in $S^{e f f}$, and they might be the key issues for making the most of Pt. 


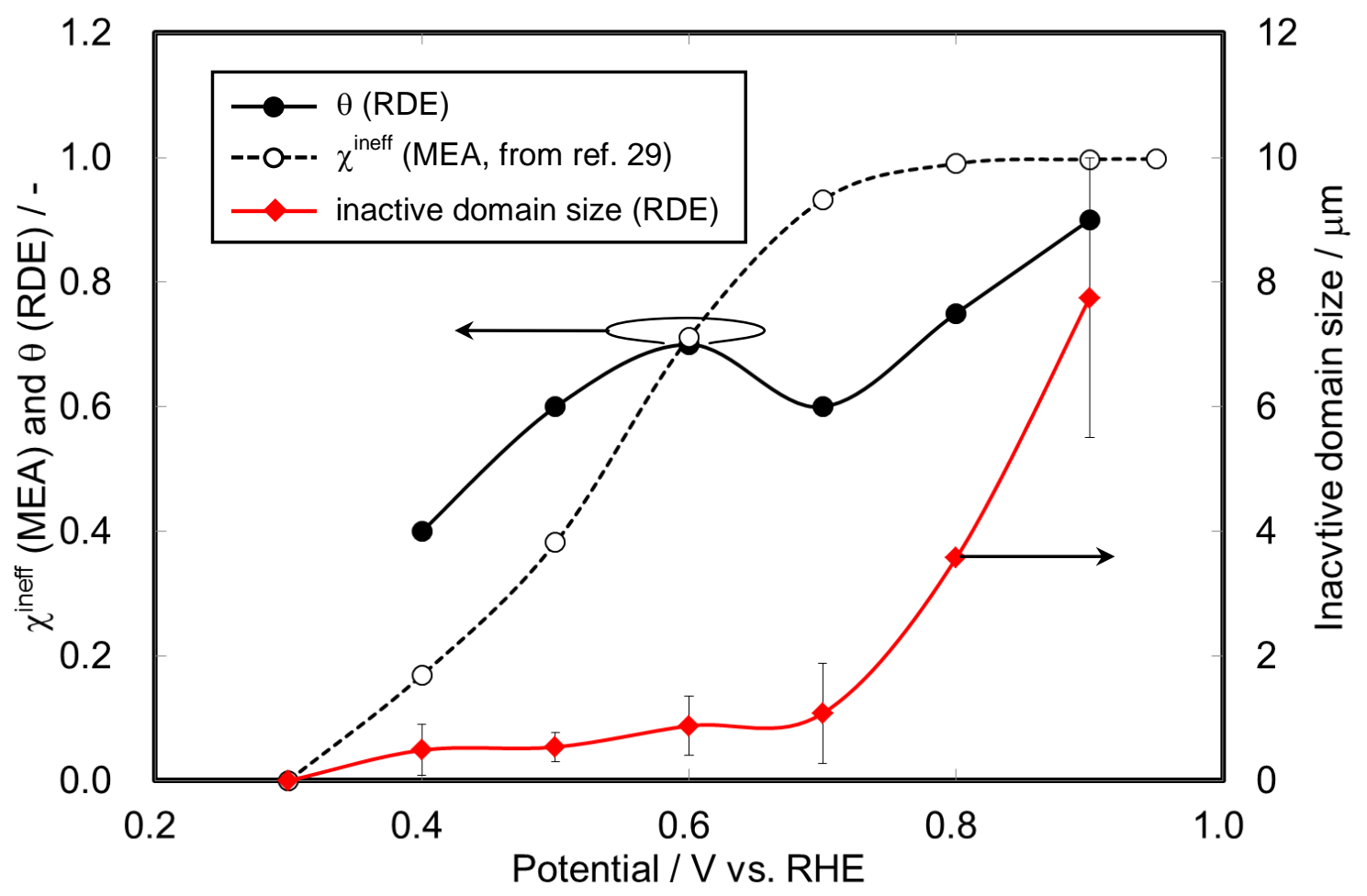

Fig. 16. $\chi^{\text {ineff }}$ (for MEA) and $\theta$ (for RDE) derived from mass transport property as a function of potential.

\section{Conclusions}

A new method for evaluating effective surface area, $S^{\text {eff }}$, for electrochemical reaction of a $\mathrm{Pt}$ disk electrode has been developed using mass transport property as a probe. Koutecky-Levich (K-L) slope for HOR was not constant and depended on both potential and rotation speed. A partial CO covering revealed that the change in K-L slope is derived from the change in $S^{\text {eff }}$. Numerical analyses based on 3 different mass transport phenomena, namely, diffusion, convection and convective-diffusion explained that the change in K-L slope expresses the transition of the mass transport phenomenon (from diffusion dominant in the low rotation region to convection dominant in the high rotation region). From the analysis, it was found possible to estimate $S^{\text {eff }}$ from the change in Koutecky-Levich slope at an extremely high rotation speed.

As $S^{\text {eff }}$ depended on both coverage, $\theta$, and inactive domain size, further analysis was necessary to quantify those parameters. Therefore, analyses with a smaller Pt disk electrode (diameter: $2 \mathrm{~mm}$ ) was done. As the coverage and the inactive domain size are probably independent of disk size, while the apparent effectiveness must depend on disk size due to convection. With the analyses using Pt disks having different diameters, it was possible to estimate $\theta$ and inactive domain size at the same time. From the comparison with $S^{\text {eff }}$ of a MEA, it was found that MEA had lower $S^{\text {eff }}$ at high potentials (over $0.6 \mathrm{~V}$ ). This is probably because the growth of Pt oxide is shifted to lower potential compared to polycrystalline platinum due to its small particle size (about $3 \mathrm{~nm}$ ).

The analysis suggests that $S^{\text {eff }}$ does decrease notably from relatively low potential (around $0.4 \mathrm{~V}$ ), even though it is not obvious in measured currents as the effect is masked by the low rates of mass transport. The analysis also suggests that there is a 
large difference in $S^{\text {eff }}$ with different methods, namely, estimation from the amount of $\mathrm{Pt}$ oxides and the analysis derived from mass transport property.

\section{Acknowledgements}

The authors would like to thank Nissan Motor Co., Ltd. for the support in financing this work and Ms. Manami Mori for her expertise in EBSD analysis.

\section{References}

[1] D. Papageorgopoulos, DOE Fuel Cells Annual Merit Review, (2012).

[2] M. Watanabe, H. Sei, P. Stonehart, J. Electroanal. Chem. 261 (1989) 375.

[3] M. Uchida, Y. Fukuoka, Y. Sugawara, N. Eda, A. Ohta, J. Electrochem. Soc. 143 (1996) 2245.

[4] R. R. Adzic, J. Zhang, K. Sasaki, M. B. Vukmirovic, M. Shao, J. X. Wang, A. U. Nilekar, M. Mavrikakis, J. A. Valerio, F. Uribe, Top. Catal. 46 (2007) 249.

[5] J. X. Wang, H. Inada, L. Wu, Y. Zhu, Y-M. Choi, P. Liu, W-P. Zhou, R. R. Adzic, J. Am. Chem. Soc. 131 (2009) 17298.

[6] F. C. Nart, W. Vielstich, in Handbook of Fuel Cells, vol. 2, Wiley, Chichester (2003) chapter 21.

[7] N. M. Markovic, H. A. Gasteiger, B. N. Grgur, P. N. Ross, J. Electroanal. Chem. 467 (1999) 157.

[8] J. X. Wang, J. Zhang, R. R. Adzic, J. Phys. Chem. A 111 (2007) 12702.

[9] N. P. Subramanian, T. A. Greszler, J. Zhang, W. Gu, R. Makharia, J. Electrochem. Soc. 159 (2012) B531.

[10] H. Iden, S. Takaichi, Y. Furuya, T. Mashi, Y. Ono, A. Ohma, J. Electroanal. Chem. 694 (2013) 37.

[11] T. J. Schmidt, H. A. Gasteiger, G. D. Stäb, P. M. Urban, D. M. Kolb, R. J. Behma, J. Electrochem. Soc. 147 (1998) 2354.

[12] E. Hguchi, H. Uchida, M. Watanabe, J. Electroanal. Chem. 583 (2005) 69.

[13] H. Yano, E. Higuchi, H. Uchida, M. Watanabe, J. Phys. Chem. B 110 (2006) 16544.

[14] C. M. Zalitis, D. Kramer, A. R. Kucernak, Phys. Chem. Chem. Phys. 15 (2013) 4329.

[15] H. Iden, A. Ohma, J. Electroanal. Chem. 693 (2013) 34.

[16] J. Maruyama, M. Inaba, K. Katakura, Z. Ogumi, and Z. Takehara, J. Electroanal. Chem. 447 (1998) 201.

[17] A. Ohma, K. Fushinobu, K. Okazaki, Electrochim. Acta 55 (2010) 8829.

[18] N. M. Marokovic, B. N. Grgur, P. N. Ross, J. Phys. Chem. B 101 (1997) 5405.

[19] N. M. Markovic, S. T. Sarraf, H. A. Gasteiger, P. N. Ross Jr., J. Chem. Soc., Faraday Trans., 92(20) (1996) 3719.

[20] J. Jiang, A. R. Kucernak, J. Electroanal. Chem. 567 (2004) 123.

[21] A. J. Bard, L. R. Faulkner, Electrochemical Methods second edition, Wiley, New York (2000) chapter 9.

[22] C.A. Angelucci, E. Herrero, J. M. Feliu, J. Phys Chem C, 114 (2010), 14154.

[23] A. R. Kucernak, G. J. Offer, Phys. Chem. Chem. Phys. 10 (2008) 3699.

[24] D. S. Strmcnik, P. Rebec, M. Gaberscek, D. Tripkovic, V. Stamenkovic, C. Lucas, N. M. Markovic, J. Phys. Chem. C 111 (2007) 18672. 
[25] N. M. Markovic, H. A. Gasteiger, P. N. Ross, J. Phys. Chem. 100 (1996) 6715.

[26] S. K. Zecevic, J. S. Wainright, M. H. Litt, S. L. Gojkovic, R. F. Savinell, J. Electrochem. Soc. 144 (1997) 2973.

[27] M. Nosek, P. Weronski, P. Nowak, J. Barbasza, Colloid and Surface A, 403 (2012) 62.

[28] V. G. Levich, Physicochemical Hydrodynamics, Englewood Cliffs, N. J., Prentice-Hall, (1962).

[29] V. Stamenkovic, T. J. Schmidt, P. N. Ross, N. M. Markovic, J. Phys. Chem. B 106 (2002) 11970.

[30] H. Iden, A. Ohma, K. Shinohara, J. Electrochem. Soc. 156 (2009) B1078.

[31] K. J. J. Mayrhofer, B. B. Blizanac, M. Arenz, V. R. Stamenkovic, P. N. Ross, N. M. Markovic, J. Phys. Chem. B 109 (2005) 14433.

[32] A. Kongkanand, J. M. Ziegelbauer, J.Phys. Chem. C 116 (2012) 3684. 